\title{
THE STRUCTURE AND THE DISTANCE OF COLLINDER 121 FROM HIPPARCOS AND PHOTOMETRY: RESOLVING THE DISCREPANCY
}

\author{
Nadia KaltcheVA \\ Department of Physics and Astronomy, University of Wisconsin Oshkosh, 800 Algoma Boulevard, \\ Oshkosh, WI 54901; kaltchev@uwosh.edu \\ AND \\ VALERI MAKAROV \\ Michelson Science Center, California Institute of Technology, 770 South Wilson Avenue, MS 100-22, \\ Pasadena, CA 91125; vvm@caltech.edu \\ Received 2007 June 15; accepted 2007 August 15; published 2007 September 19
}

\begin{abstract}
We present further arguments that the Hipparcos parallaxes for some of the clusters and associations represented in the Hipparcos catalog should be used with caution in the study of the Galactic structure. It has already been shown that the discrepancy between the Hipparcos and ground-based parallaxes for several clusters including the Pleiades, Coma Ber, and NGC 6231 can be resolved by recomputing the Hipparcos astrometric solutions with an improved algorithm diminishing correlated errors in the attitude parameters. Here we present new parallaxes obtained with this algorithm for another group of stars with discrepant data - the galactic cluster $\mathrm{Cr}$ 121. The original Hipparcos parallaxes led de Zeeuw et al. to conclude that $\mathrm{Cr} 121$ and the surrounding association of OB stars form a relatively compact and coherent moving group at a distance of $\simeq 550-600 \mathrm{pc}$. Our corrected parallaxes reveal a different spatial distribution of young stellar populace in this area. Both the cluster $\mathrm{Cr} 121$ and the extended $\mathrm{OB}$ association are considerably more distant $(750-1000 \mathrm{pc})$, and the latter has a large depth probably extending beyond $1 \mathrm{kpc}$. Therefore, not only are the recalculated parallaxes in complete agreement with the photometric $u v b y \beta$ parallaxes, but the structure of the field they reveal is no longer in discrepancy with that found by the photometric method.
\end{abstract}

Subject headings: Galaxy: structure — open clusters and associations: individual (Collinder 121) — stars: distances

\section{INTRODUCTION}

Obtaining reliable knowledge about the structure and distance of nearby $\mathrm{OB}$ associations plays a critical role in the overall study of the Milky Way morphology near the Sun. Unlike the external galaxies where the star-forming fields are generally evident from direct imaging, the study of the spiral structure of our own Galaxy is largely grounded in distance determinations of young stellar tracers. At present, sufficiently accurate astrometric data (parallaxes and proper motions) are available for few star-forming regions within $\simeq 500 \mathrm{pc}$. More comprehensive and representative studies of the local history and dynamics of star formation have to rely on the photometric method of distance determination and stellar evolution theory.

The completion of the Hipparcos catalog (ESA 1997) offered a possibility for a major improvement of the membership of young moving groups near the Sun and refining the distance scale to nearby open clusters and $\mathrm{OB}$ associations. However, the mean Hipparcos parallaxes for some galactic clusters are in disagreement with ground-based determinations by various methods. Statistically significant discrepancies between the Hipparcos trigonometric and traditional photometric, spectroscopic, and interferometric results have been reported in the literature for selected small-scale fields, most notably for the Pleiades open cluster (Pinsonneault et al. 1998; Soderblom et al. 1998, 2005; Narayanan \& Gould 1999; Stello \& Nissen 2001; Makarov 2002; Pan et al. 2004; Percival et al. 2005). Platais et al. (2007) found a similar offset in the Hipparcos mean parallax for the young open cluster IC 2391. A discrepancy was reported by Kaltcheva et al. (2005) for the open cluster IC 2602 as well. The cause for these incon- sistencies is most likely due to a faulty data reduction algorithm used in Hipparcos, which allowed highly correlated errors of along-scan attitude parameters to propagate into the fitted astrometric parameters. An alternative data reduction approach has been suggested and successfully tested by Makarov $(2002,2003)$.

The region of $\mathrm{Cr} 121$ is another example of this discrepancy. Since the discovery of a compact group at $l, b=$ $\left(234.98^{\circ},-10.21^{\circ}\right)$ by Collinder $(1931)$, both the cluster and the larger $10^{\circ} \times 10^{\circ}$ field have been extensively studied by $\mathrm{UBV}$ and $u v b y \beta$ photometry. This area includes one of the $12 \mathrm{OB}$ associations within $1 \mathrm{kpc}$ of the Sun with fairly detailed kinematical information and membership determined from Hipparcos. The Hipparcos proper motions reveal a moving group of 103 stars between $l=227^{\circ}$ and $245^{\circ}$, identifying the compact cluster $\mathrm{Cr} 121$ with an unbound extended OB association at a distance of $592 \pm 28 \mathrm{pc}$, similar to Sco OB2 (de Zeeuw et al. 1999). Robichon et al. (1999) selected 13 Hipparcos members of $\mathrm{Cr} 121$ and found a mean Hipparcos parallax of $1.80 \pm 0.24$ mas $(556 \pm 74 \mathrm{pc})$. In contrast to these results from Hipparcos, the latest $u v b y \beta$ photometric study (Kaltcheva 2000) concluded that a compact stellar group apparently identical to the genuine cluster $(\mathrm{Cr} 121)$ is situated at $1085 \pm 41 \mathrm{pc}$, and the closest members of the loose association are found at an average distance of $660-730 \mathrm{pc}$, in agreement with most of the previous photometric investigations. Since the $u v b y \beta$ photometry is arguably the best photometric system in use to provide accurate photometric distances, the origin of the discrepancy was suggested to be in the Hipparcos parallaxes for the Cr 121 members. Burningham et al. (2003) studied the low-mass pre-main-se- 
quence stars toward $\mathrm{Cr} 121$ and also came to conclusions consistent with the photometric distance determinations.

In this Letter, we consider a sample of probable members of the extended association around $\mathrm{Cr} 121$ selected by de Zeeuw et al. (1999) for which accurate $u v b y \beta$ photometry is available. The astrometric parameters of these stars are recomputed from the Hipparcos Intermediate Astrometry Data by the method proposed by Makarov (2002). The recomputed parallaxes allow us to resolve the controversy about the distance and dimensions of the $\mathrm{OB}$ association in this field.

\section{RESULTS AND DISCUSSION}

Our sample contains all 44 early-type stars with Hipparcos parallaxes listed by de Zeeuw et al. (1999) as probable members of the $\mathrm{Cr} 121$ moving group for which $u v b y \beta$ photometry is available. Homogeneous photometric $u v b y \beta$ distances are calculated for 43 of them (Kaltcheva 2000). Table 1 presents the sample, where the Hipparcos identification numbers are given in the first column, followed by the Hipparcos parallaxes and their errors, recalculated parallaxes and their errors, photometric $u v b y \beta$ distances, and MK spectral classification. The stars are formally divided into field stars (or possible association members), spread over a $10^{\circ} \times 10^{\circ}$ area around the center of $\mathrm{Cr} 121$ and six photometrically selected members of the dense cluster $\mathrm{Cr} 121$ (Kaltcheva 2000 and references therein). As follows from the data in Table 1 there is a statistically significant difference between the mean Hipparcos parallax of $1.87 \pm 0.15$ mas and the mean recomputed parallax of $1.29 \pm 0.15$ mas. The errors provided here are the formal standard deviation of the mean computed from the formal errors of parallaxes.

Figure 1 shows the original Hipparcos parallaxes (left plot) and our recomputed parallaxes (right plot) versus the photometric parallaxes for the sample of 43 stars in Table 1 . The Hipparcos parallaxes are on average larger than the photometric values by $0.52 \pm 0.107$ mas, where the quoted error is the sample standard error of the mean. This is a statistically significant difference of the same order as those found for the Pleiades and a few other Galactic clusters. On the other hand, the agreement is excellent between the mean photometric parallax and the mean corrected parallax $(0.063 \pm$ 0.158 mas). This supports our main conclusion that the Hipparcos parallaxes are systematically overestimated in this area of the sky. But Figure 1 also reveals another strange property of the original parallaxes. While the recomputed parallaxes are scattered fairly symmetrically around the line of unit slope in the right plot and their dispersion is in good agreement with the measurement errors, the original parallaxes are grouped tightly around the mean (1.87 mas) with a standard deviation of only 0.61 mas. This value is much too small for the estimated formal errors (mean 0.93 mas, rms 0.96 mas). We attribute this result to a strong selection effect in the method employed by de Zeeuw et al. (1999), which preferentially accepted stars with large measured parallaxes, i.e., mostly stars with positive errors "observed minus true." In combination with the correlated error of the mean parallax, this selection bias gives rise to doubt about the completeness and reliability of the present membership list.

The significant dispersion of both photometric and recomputed parallaxes also implies a complex morphology of this moving group having a considerable depth, as opposed to the previous conjecture of an association compressed in the radial dimension, similar to the nearby Sco OB2 association, as concluded by de Zeeuw et al. (1999). The group also appears
TABLE 1

THE SAMPLE

\begin{tabular}{|c|c|c|c|c|c|c|}
\hline HIP & $\begin{array}{c}\pi \\
\text { (mas) }\end{array}$ & $\begin{array}{c}\sigma_{\pi} \\
(\mathrm{mas})\end{array}$ & $\begin{array}{c}\pi_{r} \\
(\mathrm{mas})\end{array}$ & $\begin{array}{c}\sigma_{\pi_{r}} \\
(\mathrm{mas})\end{array}$ & $\begin{array}{c}r \\
(\mathrm{pc})\end{array}$ & MK \\
\hline \multicolumn{7}{|c|}{ Field Stars } \\
\hline 31436 & 1.14 & 0.90 & 0.67 & 0.94 & 1812 & $\mathrm{~B} 2 / \mathrm{B} 3 \mathrm{~V}$ \\
\hline 31901 & 2.05 & 1.06 & 0.95 & 1.07 & 1050 & B5 \\
\hline 32084 & 2.63 & 1.18 & -1.02 & 1.09 & 664 & B3 V \\
\hline 32101 & 1.24 & 1.07 & -0.21 & 1.11 & 938 & B9.5 III \\
\hline 32591 & 1.42 & 0.97 & 0.14 & 0.98 & 605 & B8 V \\
\hline 33007 & 1.16 & 0.86 & 1.17 & 0.92 & 475 & B4 V \\
\hline 33092 & 2.02 & 0.70 & 2.37 & 0.78 & 518 & B1 Ib \\
\hline 33165 & 1.74 & 0.76 & 1.33 & 0.85 & $\ldots$ & WN... \\
\hline 33260 & 1.19 & 1.10 & 0.98 & 1.11 & 930 & B9 Ib/II \\
\hline 33294 & 1.43 & 0.69 & 0.82 & 0.77 & 681 & B2 III/IV \\
\hline 33316 & 1.51 & 0.64 & -0.32 & 0.73 & 632 & B2/B3 III \\
\hline 33447 & 2.78 & 0.70 & 1.23 & 0.77 & 766 & B2 III/IV \\
\hline 33523 & 1.70 & 1.23 & -0.41 & 1.25 & 1697 & B2 V \\
\hline 33532 & 2.24 & 0.73 & 1.04 & 0.85 & 539 & B2.5 III \\
\hline 33611 & 2.05 & 0.70 & 1.40 & 0.76 & 722 & $\mathrm{~B} 2 \mathrm{~V}$ \\
\hline 33621 & 1.70 & 0.93 & 0.29 & 0.98 & 764 & B8 II/III \\
\hline 33666 & 2.33 & 0.68 & 0.90 & 0.76 & 740 & B2 III \\
\hline 33673 & 1.68 & 0.72 & 0.56 & 0.78 & 923 & B4 Vn \\
\hline 33721 & 2.46 & 0.74 & 1.43 & 0.81 & 706 & B3 Vnn \\
\hline 33769 & 1.26 & 0.80 & 0.25 & 0.85 & 1077 & $\mathrm{~B} 2 / \mathrm{B} 3 \mathrm{~V}$ \\
\hline 33770 & 2.05 & 0.97 & 1.31 & 1.28 & 630 & B2 IV \\
\hline 33804 & 3.17 & 0.59 & 3.29 & 0.66 & 365 & B2/B3 III/IV \\
\hline 33814 & 2.31 & 0.93 & 2.44 & 0.97 & 887 & B3 V \\
\hline 33846 & 1.41 & 0.74 & 2.04 & 0.80 & 647 & B3 V \\
\hline 33865 & 1.75 & 1.18 & -0.14 & 1.35 & 648 & B3 IV \\
\hline 33888 & 1.35 & 1.13 & 1.38 & 1.14 & 793 & B9 $V+\ldots$ \\
\hline 34041 & 1.79 & 0.66 & 1.48 & 0.72 & 521 & $\mathrm{~B} 2 / \mathrm{B} 3 \mathrm{~V}$ \\
\hline 34067 & 1.66 & 0.80 & 2.27 & 0.84 & 853 & B3 III \\
\hline 34074 & 1.10 & 1.10 & 1.82 & 1.13 & 1597 & B7/B8 III \\
\hline 34153 & 2.55 & 1.06 & 1.92 & 1.09 & 535 & B8 V \\
\hline 34167 & 1.44 & 0.91 & 1.45 & 0.94 & 958 & B2 IV \\
\hline 34219 & 1.95 & 1.67 & 2.94 & 1.38 & 665 & B6 III \\
\hline 34227 & 1.04 & 0.94 & 0.90 & 0.97 & 757 & B3 V:n \\
\hline 34281 & 1.28 & 1.03 & 1.16 & 1.04 & 842 & B5 V \\
\hline 34331 & 2.23 & 0.65 & 1.11 & 0.71 & 534 & B2 IV-V \\
\hline 34579 & 1.78 & 0.60 & 1.64 & 0.68 & 368 & $\mathrm{~B} 2 \mathrm{~V}$ \\
\hline 34940 & 2.07 & 1.24 & 3.18 & 0.98 & 676 & B2 IV \\
\hline 35026 & 1.44 & 0.78 & 2.34 & 0.83 & 1435 & B2 IV/V \\
\hline \multicolumn{7}{|c|}{ Cr 121} \\
\hline 32823 & 1.92 & 1.23 & 2.41 & 1.26 & 944 & B5 V \\
\hline 32911 & 3.49 & 1.02 & 2.75 & 1.05 & 1012 & B8 IV/V \\
\hline 33062 & 1.22 & 0.96 & 0.81 & 1.02 & 947 & B2 II/III \\
\hline 33070 & 2.30 & 1.13 & 0.97 & 1.17 & 1291 & B3 II/III \\
\hline 33208 & 1.77 & 1.14 & 1.16 & 1.16 & 981 & B3 V \\
\hline 33211 & 3.46 & 1.08 & 2.51 & 1.11 & 1131 & B3 V \\
\hline
\end{tabular}

Note. - Identifications from the Hipparcos catalog, followed by the Hipparcos parallaxes $\pi$ and their formal errors $\sigma_{\pi}$, the recalculated parallaxes $\pi_{r}$ and their formal errors $\sigma_{\pi_{r}}$, the photometric $u v b y \beta$ distances $r$, and the MK classification.

to be located at a larger distance of $\simeq 740 \mathrm{pc}$, rather than at $\simeq 550 \mathrm{pc}$ as follows from the mean Hipparcos parallax of the sample in Table 1. Based on a larger photometric sample, it has already been pointed out that the loose nearby structure defined by de Zeeuw et al. (1999) to be located at $592 \pm$ $28 \mathrm{pc}$ photometrically appears to be more distant by about 100 pc (Kaltcheva 2000). The parallaxes recalculated here support the photometric findings.

Our result implies that the problem of inaccurate mean parallaxes in Hipparcos affects more regions, and of larger angular area, than just a few small patches occupied by dense open clusters. This is not an irreversible situation, because the method of astrometric solution of the available Hipparcos data used in this Letter proves once again successful in correcting this error, despite its limitations. A more systematic and thorough comparison of Hipparcos data with distances 

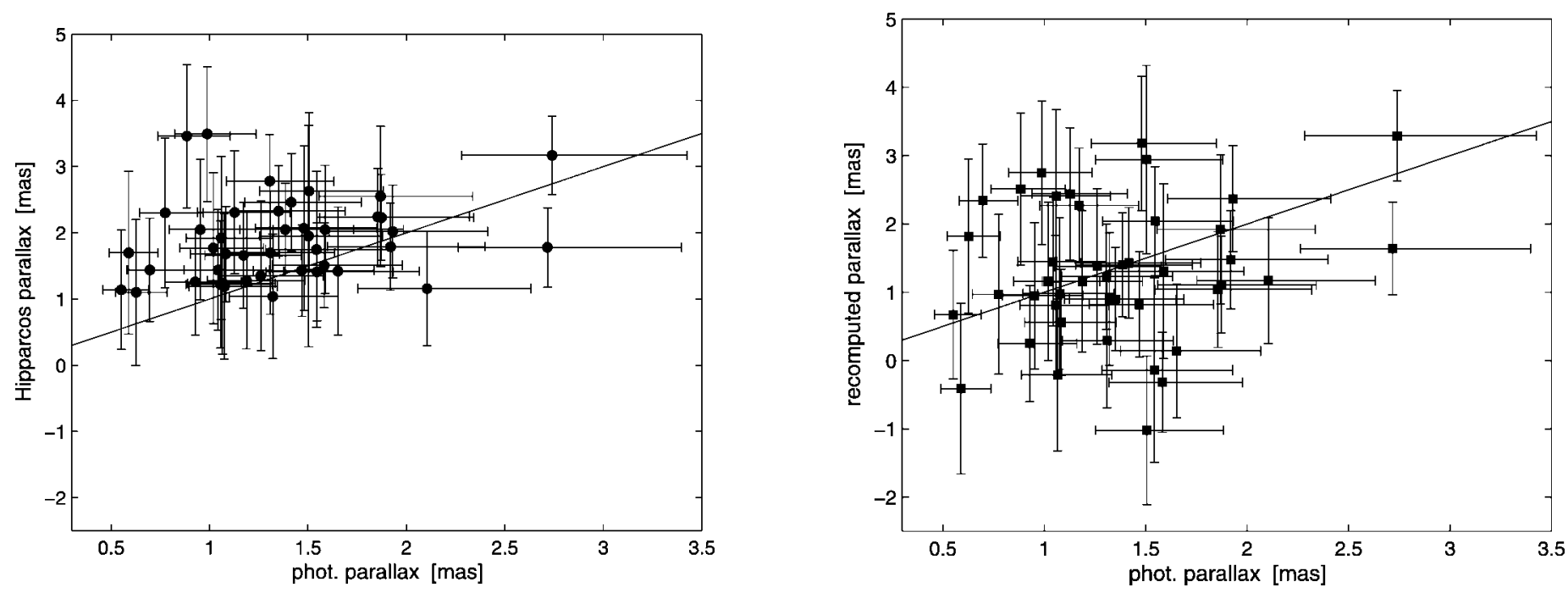

Fig. 1.-Differences between the uvby $\beta$ photometric parallaxes and Hipparcos parallaxes (left plot), and the parallaxes recomputed in this Letter (right plot) for stars in the area of $\mathrm{Cr} 121$. The error bars of the photometric parallaxes correspond to the maximum estimated error in the photometric distances of $20 \%$.

from precision multiband photometry will probably reveal more problematic areas. It is not clear at present how widely spread the parallax error is, and whether a global astrometric solution will have a significant impact on the present knowledge of distances and morphology for many of the OB associations represented in the catalog, but it is evident that the Hipparcos-based census of some of the moving groups near the Sun should be critically reconsidered.
We are grateful to the referee R. Hanson for a number of valuable comments. A UW Oshkosh Vander Putten Award is acknowledged. Partial support was provided by the National Science Foundation under grant AST-0708950 to N. K. The research described in this Letter was in part carried out at the Jet Propulsion Laboratory, California Institute of Technology, under a contract with the National Aeronautics and Space Administration.

\section{REFERENCES}

Burningham, B., Naylor, T., Jeffries, R. D., \& Devey, C. R. 2003, MNRAS, 346,1143

Collinder, P. 1931, Ann. Obs. Lund, 2, 1

de Zeeuw, P. T., Hoogerwerf, R., de Bruijne, J. H. J., Brown, A. G. A., \& Blaauw, A. 1999, AJ, 117, 354

ESA. 1997, The Hipparcos Catalogue, Vol. 1 (ESA SP-1200; Noordwijk: ESA) Kaltcheva, N. T. 2000, MNRAS, 318, 1023

Kaltcheva, N. T., Jaeger, S., Kaba Bah, M., \& Briley, M. M. 2005, Astron. Nachr., 326, 738

Makarov, V. V. 2002, AJ, 124, 3299

. 2003, AJ, 126, 2408

Naryanan, V. K., \& Gould, A. 1999, ApJ, 523, 328
Pan, X., Shao, M., \& Kulkarni, S. R. 2004, Nature, 427, 326

Percival, S. M., Salaris, M., \& Groenewegen, M. A. T. 2005, A\&A, 429, 887 Pinsonneault, M. H., Stauffer, J., Soderblom, D. R., King, J. R., \& Hanson, R. B. 1998, ApJ, 504, 170

Platais, I., Melo, C., Mermilliod, J.-C., Kozhurina-Platais, V., Fulbright,J. P.,

Mendez, R. A., Altmann, M., \& Sperauskas, J. 2007, A\&A, 461, 509

Robichon, N., Arenou, F., Mermilliod, J. C., \& Turon, C. 1999, A\&A, 345, 471

Soderblom, D. R., King, J. R., Hanson, R. B., Jones, B. F., Fisher, D., Stauffer, J. R., \& Pinsonneault, M. 1998, ApJ, 504, 192

Soderblom, D. R., Nelan, E., Benedict, G. F., McArthur, B., Ramirez, I., Spiesman, W., \& Jones, B. F. 2005, AJ, 129, 1616

Stello, D., \& Nissen, P. E. 2001, A\&A, 374, 105 